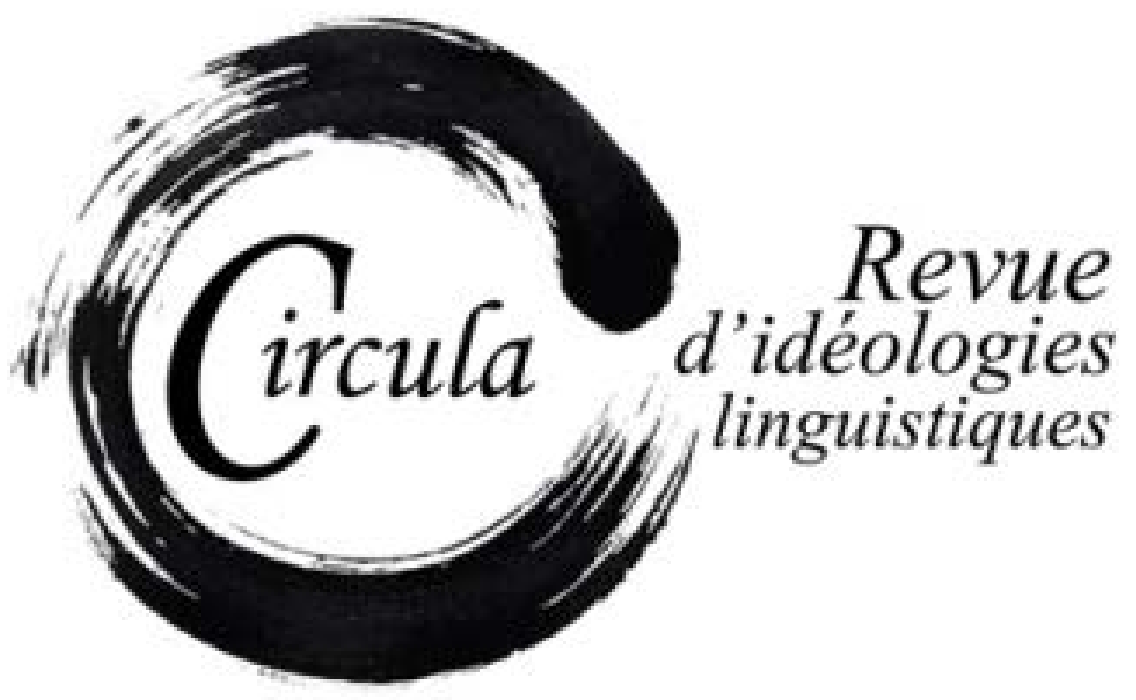

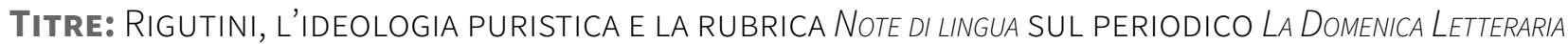
Auteur(s): VAlentina AlLiA, UniVersitì di Messina

Revue: Circula, NumÉRO 5

PAgES: $25-41$

ISSN: 2369-6761

DiRecteurs: Wim Remysen, Sabine SchWARze et JuAn Antonio EnNis

URI: HTTP://HDL.HANDLE.NET/11143/11231

DOI: HTTPS://DOI.ORG/10.17118/11143/11231 


\section{Rigutini, l'ideologia puristica e la rubrica Note di lingua sul periodico La Domenica Letteraria}

Valentina Allia, Università di Messina alliavalentina@ alice.it

Riassunto: Questo contributo si propone di analizzare alcuni articoli scritti da Giuseppe Rigutini nella rubrica Note di lingua, apparsa sul periodico La Domenica Letteraria tra il 1882 e il 1883. In quanto nucleo costitutivo originario del volume I Neologismi buoni e cattivi più frequenti nell'uso odierno, la rubrica riveste particolare importanza per ricostruire i dubbi dei parlanti del tempo e la loro coscienza linguistica. Tra le questioni affrontate spiccano quelle lessicali, che riguardano principalmente la diffusione e la traducibilità di alcuni forestierismi, in particolare francesismi, sempre più infiltrati nelle maglie dell'italiano. Scopo di questa ricerca è ricostruire l'ideologia linguistica di Rigutini a partire dalle domande poste dai lettori della rubrica e inquadrare la sua figura in relazione all'ideologia puristica.

Parole chiave: giornali; storia della lingua italiana; stranierismi; purismo

Abstract: This paper investigates some articles written by Giuseppe Rigutini in the column Note di lingua and published in the magazine La Domenica Letteraria from 1882 to 1883. These articles were part of the prescriptive book / Neologismi buoni e cattivi piu frequenti nell'uso odierno and they comment contemporary language use, such as the prevalence and translatability of some foreign words in Italian. Rigutini's column gathers information on doubts and misgivings about ancient and new words, in particular French words, and about the correct use of Italian language. This research sheds light on Rigutini's linguistic ideology and his relationship with purism and pays particular attention to lexical issues that interested him.

Keywords: magazines; history of Italian language; foreign words; purism 


\section{Introduzione}

La rubrica Note di lingua a firma di Giuseppe Rigutini apparve per la prima volta sul periodico La Domenica Letteraria, diretto da Ferdinando Martini, nel marzo del 1882 e andò avanti, a singhiozzo', fino al settembre del 1883.

Secondo il progetto iniziale, le Note si sarebbero dovute raccogliere successivamente in un volume curato da Rigutini e da Martini. Siccome sarebbero occorsi molti anni per mettere insieme il volume, Rigutini decise di cessare le Note e di scrivere in un libro - e cioè, i Neologismi buoni e cattivi più frequenti nell'uso odierno - «brevi appunti su quanto rimaneva ancora di vocaboli, maniere e usi da meritare di essere, al mio giudizio, o difesi o condannati» (Rigutini, 1886)². Nell'Avvertimento alla prima edizione dei Neologismi del 1886, Rigutini afferma che il volume fu completato già nel 1884 ma le vicende dell'editore ne ritardarono per due anni la pubblicazione.

Al fine di delineare la vicenda editoriale della rubrica, sono state confrontate le edizioni dei Neologismi del 1886, del 1905, del $1912^{3}$ e l'ultima del 1926, in larga parte uguale alle due immediatamente precedenti, ma con aggiunte del manzoniano Giulio Cappuccini apposte in forma di dizionario supplementare alla fine del volume.

In quanto nucleo costitutivo originario dell'opera, infatti, l'autore riportò nei Neologismi le Note di lingua pubblicate sul periodico, ponendole - nell'edizione del 1886 - nella prima parte del volume, salvo pentirsi successivamente di questa scelta e auspicare una collocazione in appendice «se il libro avrà la fortuna di una ristampa»4: è ciò che avvenne nelle edizioni del 1905 (quinta edizione) e del 1912 (sesta edizione), nelle quali alcune Note sono state anche impiegate come descrizione dei lemmi corrispondenti ${ }^{5}$. Nell'edizione di Cappuccini, invece, le Note di lingua non figurano.

1. In Note di Lingua (d'ora in poi NDL), a. II, n’ 11, 18 marzo 1883, Rigutini esordisce così: «Riprendo le mie note di lingua, sollecitato da molti fra i lettori della Domenica Letteraria, e che io ho da qualche tempo trascurate, dubitando invece di riescir molesto». Insieme ad importanti personalità della seconda metà del XIX secolo, Rigutini collaborò anche ad un progetto editoriale volto a dedicare attenzione all'educazione femminile: scrisse sulle pagine della rivista Cordelia (De Roberto, 2016: 102). Del «moderato e manzoniano Rigutini [...] redattore della rubrica Note di lingua nella "Domenica Letteraria" di Ferdinando Martini» scrive Gatta (2014: 323).

2. L'Avvertimento premesso all'edizione 1886 dei Neologismi buoni e cattivi più frequenti nell'uso odierno differisce in pochi luoghi con quello delle edizioni successive.

3. Le edizioni del 1905 e del 1912 sono sostanzialmente identiche. Da ora in poi i riferimenti e le citazioni dall'edizione del 1912 saranno implicitamente validi anche per quella del 1905.

4. Si cita dall'Avvertimento premesso all'edizione del 1886, in cui Rigutini scrive: «Soprattutto non mi piacque la divisione in due parti, che allora mi fu imposta dalla necessità, e meglio sarebbe stato che quelle Note, le quali compongono la prima, fossero incorporate nella seconda. Tutto questo sarà fatto, se il libro avrà la fortuna di una ristampa» (Rigutini, 1886). Nell'Avvertimento dell'edizione del 1912, infatti, scrive cosi: «Tutto ciò ho fatto in questa ristampa, mettendo in Appendice quello che non poteva stare nel corpo del libro» (Rigutini, 1912: VI).

5. Nel corso di questo lavoro darò conto del riuso di alcune voci o questioni linguistiche trattate nella rubrica nelle varie edizioni dei Neologismi. Le Note analizzate sono presenti nella prima edizione del 1886, salvo casi debitamente segnalati. 
Come si legge nell'Avvertimento all'edizione del 1912, le Note sono depositarie di alcuni principi seguiti dal lessicografo e, quindi, risultano utili a noi per inquadrare meglio la sua figura e la sua ideologia linguistica. Va subito precisato che Rigutini non è un purista in senso storico: anzi, non mancano esplicite dichiarazioni in cui si schiera apertamente contro i puristi6: li definisce con intento ironico e spregiativo «venerandi antiquarj della lingua», e dichiara che «sarebbe poco glorioso combattere coi moribondi» ${ }^{7}$. È pur vero che è prassi consolidata, nella storia della lingua, rigettare questa etichetta: persino Fanfani e Arlia dichiarano di non essere puristi ${ }^{8}$. Va detto che l'adesione all'ideologia puristica si manifesta in modi e forme diverse a seconda delle varie personalità e dei frangenti storici: nella nostra indagine cercheremo i segni di un'adesione o di un distacco da parte del nostro da posizioni genericamente ascrivibili al purismo ottocentesco.

\section{La riflessione linguistica sulle colonne del periodico}

Il Rigutini accetta l'incarico di scrivere le Note rivolgendosi al Martini in questi termini:

Eccoti dunque, mio caro Martini, questo Prefazio a quelle brevissime Note di lingua, che tu vuoi ch'io faccia via via nella Domenica Letteraria; eccoti in poche parole i criterj ch'io seguirò, non senza però tacerti che mi hai dato una gatta a pelare, e non senz'anche dichiarare a tutti i lettori del tuo periodico, ch'io, come non intendo di montar sul tripode e dettar responsi, così non ho alcuna voglia di attaccar brighe con nessuno, rispondendo con un comodo e... dignitoso silenzio a chiunque vorrà contraddirmi. (Rigutini, 1886: 14)

In realtà, in qualche puntata della rubrica abbandona questa posizione di altero distacco e si difende veementemente dall'accusa di aver plagiato il Dizionario di Fanfani nel suo Vocabolario della Lingua italiana per le scuole , pur ammettendo con umiltà la possibilità di aver commesso errori nella compilazione. Il dichiarato ossequio alla Crusca del Manuzzi ${ }^{10}$, fedele allievo del Cesari, suona come un suggerimento prescrittivo e passatista, che ricollega il lessicografo a posizioni proprie dei puristi.

6. Nell'Avvertimento all'edizione 1886 Rigutini scrive: «Ho preso poi a combattere il Lessico dell'infima e corrotta italianità, parendomi un libro più dannoso che utile agli studi» (Rigutini, 1886).

7. Cf. Si dice o non si dice? Lettura fatta al circolo filologico fiorentino, in cui il lessicografo esprime, tra l'altro, il suo interessante punto di vista su questioni linguistiche importanti del periodo (Rigutini, 1912: XIV).

8. È stata più volte evidenziata l'avversione per l'etichetta di purista da parte di Fanfani e Arlìa (Serianni, 2013: 79; Vitale, 1986: 548).

9. Il riferimento è al Vocabolario della lingua italiana compilato per uso delle scuole accresciuto di voci del comun parlare e degli approvati scrittori, Firenze, Barbera, 1879.

10. A questo proposito, è bene ricordare che «Manuzzi fu un purista come Cesari, e il suo vocabolario, per quanto corretto e accurato, conferma la tendenza di una parte della cultura italiana ad assestarsi nel solco del passato, a radicarsi in esso» (Marazzini, 2002: 385). 
Riporto qui di seguito ciò che scrive Rigutini nella Nota dal titolo Lettera di un plagiario al Direttore della Domenica Letteraria ${ }^{11}$, apparsa esclusivamente sul periodico e non ripubblicata in volume:

Mio caro direttore ${ }^{12}$,

Questa volta, invece d'una Nota, ti mando una lettera che ti prego in cortesia tu voglia pubblicare nella Domenica Letteraria. Ė forse un po' troppo lunga; ma al mio bisogno è certamente troppo breve: tuttavia non abuserò né del tuo giornale, né della pazienza de' tuoi lettori.

Finché per più di tre anni io ebbi dinanzi a me un tale, che non degno nominare, credetti provvedere al mio decoro non rispondendo alle malevole accuse ed alle censure che periodicamente, e massimo all'avvicinarsi della primavera, mi va regalando ne' suoi libercoli, che nessuno legge o pochissimi. Ma ora che le accuse sue vanno da taluno ripetendosi in qualche giornale anche politico e si cerca di formare artificiosamente contro di me una falsa opinione, io non posso né debbo più tacere; e per la prima volta, che sarà anche l'ultima, mi risolvo a rispondere.

L'accusa formulata ora contro di me da quel mio accusatore è questa: che io compilando per il Barbèra il Vocabolario della Lingua italiana per le Scuole, non ho fatto altro che commettere un plagio, copiando quello di P. Fanfani, o solo aggiungendo di mio qualche sproposito. A questa accusa, grave abbastanza, come ognuno capisce, si vuol dare apparenza di verità con recare innanzi alcune definizioni prese da due Vocabolari, mostrandone l'identità. [...] lo ho dato il modo per chi voglia seguitare da sé nel confronto, e qui torno a raccomandare che nel confronto si abbia sempre innanzi la Crusca, specie quella del Manuzzi. Quanto poi a certi errori di definizioni, in cui posso esser caduto, anche condottovi dall'autorità del F., io non vorrò negarli; i quali spariranno se, come Pietro Fanfani trovò nel suo editore, così io troverò nel mio la cura di ristampare questo Vocabolario. Dicendo ciò non creda il mio censore che io dia gran peso alle censure contenute nel suo libercolo, il quale, come tutti gli altri libercoli suoi di questo genere, si compono [sic] di asinità, di presunzione, di pedanteria, di scioccheria, di mal animo e di un poco, ma ben poco, di ragione. Del resto egli è oramai avvezzo a fare il sopraccio in queste materie, e la sua focula si esercita indifferentemente sulle spalle della Crusca, del Tortoli o sulle mie. (NDL, a. II, n’ 20, 20 maggio 1883)

11. Si tratta di una Nota che, per il tono e la sostanza concettuale affrontata, si presta meglio ad essere accolta sulle colonne del periodico e non in volume: come già evidenziato sopra, non c'è traccia della questione nelle varie edizioni dei Neologismi.

12. In tutte le citazioni di questo lavoro il corsivo è dell'autore. 


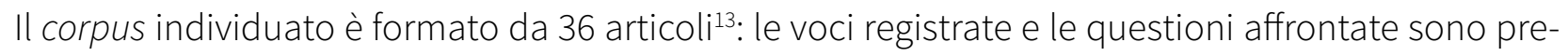
sentate talvolta come semplici riflessioni linguistiche, ma spesso appaiono in forma di lettere, reali o finte, di processi, di ricorsi, di aneddoti, come avviene anche nel Lessico di Fanfani e Arlia (Serianni, 2013: 79).

\subsection{Francesismi}

Da uno sguardo complessivo alle questioni linguistiche trattate nella rubrica si evince che un consistente numero di interventi si incentra sul rifiuto, tipicamente puristico, dei francesismi.

Nella seconda metà del XIX secolo, tra le lingue straniere, il francese mantiene intatto il suo primato e l'inglese filtra perlopiù attraverso traduzioni mediate dal francese: Rigutini fotografa questa situazione in una Nota in cui rimprovera certi «scerpelloni di grammatica» e l'uso di «voci del ributtantissimo gergo italo-gallo» ai traduttori dei romanzi di Giovanni Ruffini, che - sebbene siano stati scritti in inglese - sono stati tradotti in italiano dal francese $e^{14}$, facendo scempio della nostra lingua e deformando il libro «con laido impasto di spropositi». Riportiamo uno stralcio dall'intervento di Rigutini:

E sfido chiunque non sia addirittura un barbaro, come il traduttore, a non sentirsi sollevar la bile, leggendo, per esempio, nel Lorenzo Benoni «Margherita metteva sotto chiave ogni avanzi». «Un abbate costumava venire al dopo pranzo d'ogni giorno ad iniziarmi nei misteri della lingua latina alla tassa di tre soldi per ora». [...] «Fra mezz'ora arrivammo alla chiesa». [...] «Comprendo benissimo come tu non sia contento dell'opera tua, anzi che tu non possa esserlo mai. La mediocrità, essa, lo è più soventi.» [...] E più avanti abbiamo mangiarsi il fatto suo, e sfumante di rabbia, e ancheggiare al Pincio, e di molto troppo astuto, e tenere il lettore sulla griglia, e sapere per core! E tante e tante altre infamie, le quali ci dimostrano chiaramente che i libri di Giovanni Ruffini non sono stati tradotti dall'inglese, ma da traduzioni francesi. (NDL, a. I, n’39, 29 ottobre 1882)

L'interesse per i romanzi di Giovanni Ruffini verrà manifestato da Rigutini ancora alcuni anni dopo, al tempo dell'edizione dei Neologismi del 1886, quando darà notizia di una sua traduzione del Lorenzo Benoni, concepita con l'aiuto di sua moglie, «versatissima nella lingua inglese», e stampata dal Trevisini (Rigutini, 1886: 90).

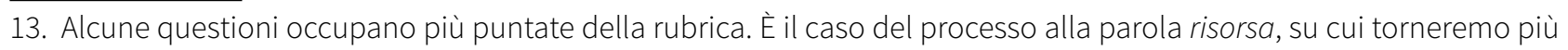
avanti.

14. Ci si riferisce a NDL, a. I, n’ 39, 29 ottobre 1882, poi ripubblicata nei Neologismi del 1886 (Rigutini, 1886: 87), ma non nelle edizioni successive. Per quanto concerne il problema delle traduzioni, va detto che «Nella seconda metà del secolo la situazione è diversa: aumentano le traduzioni basate direttamente sull'originale, in particolare per opere inglesi (ma i romanzi russi, "di gran moda nel nostro Occidente", come annotava il Panzini, vengono generalmente tradotti dal francese), e proliferano le versioni popolari pubblicate a puntate sui giornali, che spesso lasciano inalterati i forestierismi meno facilmente traducibili (più per la frettolosità della confezione che per deliberata scelta espressiva)» (Serianni, 2013: 158). 
L'idea che viene veicolata attraverso le Note rigutiniane è che i francesismi sono ben accetti soltanto quando sono veramente necessari o rispettano i meccanismi di formazione dell'italiano. Nella lettera di prefazione al Martini, contenuta nella prima edizione dei Neologismi, infatti, si leggono le ideologie linguistiche già rintracciabili nella sua rubrica giornalistica:

io accetto, fosse anche turca, ogni nuova parola che la mia lingua non mi dà né mi può dare, e che è necessaria a significare qualche cosa di nuovo. Gl'Inglesi, ad esempio, che pur sono gelosi dell'idioma loro, non fanno altrimenti. Né è da scalmanarsi tanto contro a tutte le nuove voci che ci vengono di Francia, posto, ripeto, che ci siano veramente necessarie; perché in generale sono derivate dal latino, che è la sorgente di nostra lingua, e quando siano improntate del vero conio italiano, non v'è ragione perché debbano essere rifiutate. (Rigutini, 1886: 13)

Nella Nota sulla voce decorazione ${ }^{15}$ scrive: «non di ogni francesismo penso dobbiamo impaurirci, ma che anzi si debba accettare tutto ciò che è ben formato e può convenire con l'indole di nostra lingua. [...] nulladimeno io non vorrei difenderla se non avesse buona radice nel latino decus»; diverso, invece, l'atteggiamento in un'altra Nota in cui bolla come inutile, superfluo e barbarico il ricorso all'avverbio l'indomani ${ }^{16}$ (sempre preceduto dall'articolo, come in francese), «maniera introdotta senza bisogno e da potersene anche far senza», come ammette lo stesso Gherardini, che, però, la difende insieme a Viani e insieme a tutti gli altri puristi permissivisti (Serianni, 2013: 66) che sono troppo «accettatori del si dice». Rigutini risponde a quelli che gli fanno notare che l'indomani viene adoperato in Toscana ammettendo che è usato «non dal popolo, né dalle persone colte che parlano toscanamente; ma da chi ha imparata la lingua nei romanzi francesi originali o tradotti, da chi dicendo l'indomani crede scioccamente distinguersi dal popolo, e da chi, senza volerlo, si lascia uscir dalla penna questa o altra sgarbatezza» (NDL, a. I, n 17, 28 maggio 1882). Tra questi ultimi, annovera anche «il severo, lo stitico Botta» che, a domande su altri gallicismi da lui adoperati, risponde sempre ringraziando chi gli fa notare di aver usato simili maniere e promette di guardarsene in futuro.

Rigutini ammette, dunque, che alcuni francesismi possono essere usati senza volerlo: è il sintomo di una infiltrazione profonda, seppure inconsapevole, nelle maglie della coscienza linguistica dei parlanti. Nell'Avvertimento all' edizione 1912 osserva con rassegnazione che tutti quelli che «si affaticano a far riparo contro l'invadente forestierume nella nostra lingua» non hanno buone speranze di riuscita poiché «ormai una corrente rapida e lutulenta travolge la lingua e il pensiero italiano; di modo che tra cinquant'anni sarà additato per cosa mirabile, come direbbe messer Francesco Petrarca, chi penserà e scriverà in vero italiano» (Rigutini, 1912: VII).

15. Per decorazione cf. NDL, a. I, n’ 14, 7 maggio 1882, che viene accolta, con qualche lieve modifica, come commento del lemma nelle edizioni successive in volume (Rigutini, 1912: 52; 1926: 52).

16. Per l'indomani cf. NDL, a. I, n 17, 28 maggio 1882, in cui Rigutini, a proposito del Viani, dice: «è troppo avvocato delle voci incriminate», salvo alleggerire il giudizio nei Neologismi del 1912 sostituendo incriminate con censurate: «difensore delle voci censurate» (Rigutini, 1912: 95). Va ricordato che Manzoni usa l'indomani nei Promessi Sposi (De Roberto, 2016: 113). 
La profezia non si è avverata, ma l'affermazione del lessicografo è una significativa constatazione della «ineluttabilità di un processo inarrestabile» (Della Valle, 1994: 74), governato dall'imporsi dell'uso: Rigutini, dunque, si avvicina a Manzoni nella consapevolezza del potere dell'uso, a cui la naturalezza della lingua non può rinunciare.

In questo senso trova ragione, in un altro numero della rubrica, la contestazione dei termini brindare e bissare ${ }^{17}$, introdotti dalla lingua dei giornali e derivanti dal francese: Rigutini li definisce

due nuovi mostriciattoli, venutoci il primo dentro una bottiglia di Sciampagna, il secondo natoci forse in qualche camerino di teatro. Ma per mostriciattoli che siano, e' son tenuti in collo e vezzeggiati specialmente da quella brava gente dei Cronisti e referendarii de' Giornali [...]. E così non si può aprir giornale, che parlando di un banchetto o di una rappresentazione melodrammatica o di un concerto, non ci metta sotto gli occhi questi due bei cosini. Ma buon Dio! Ė forse peccare di pedanteria se dico che invece di brindare (dal francese brindé) sarebbe più decente continuare a tenerci il cristiano bevere alla salute, alla prosperità, all'incremento e che altro si voglia di una persona, o di una cosa; o fare un brindisi oppure se piaccia più solenne vocabolo propinare; e invece di bissare, parola che sente del solito gergo teatrale, i signori Cronisti volessero contentarsi del vecchio Richiedere? (NDL, a. I, n’ 12, 23 aprile 1882)

Quella che il lessicografo pone è, dunque, una questione di gusto: nella stessa nota dirà, poche righe più avanti, che gli riesce difficile rispondere ad alcune domande giunte in redazione «perché concernenti certi parlari figurati che bisogna lasciare al gusto, corretto e scorretto, di chi le adopra». Quando un lettore di Roma lo interpella a proposito della bontà dell'espressione brandire i calici, risponde:

se io dovessi brandire un calice per romperlo nella testa a qualcuno, non mi parrebbe di dire (non dico di fare) uno sproposito: ma se volessi brandirlo per fare un brindisi e poi vuotarlo, a me parrebbe che il brando facesse in questa figura una figura meschina. Ma queste, ripeto, son cose di gusto, et de gustibus con quel che segue. (NDL, a. I, n 12, 23 aprile 1882)

17. Per brindare e bissare, nelle edizioni 1912 e 1926, si legge un commento più sintetico: «Nei troppo frequenti banchetti politici è raro che non si oda questa voce, e che i gazzettieri non la ripetano. Ma perché escludere l'italiano Bevere o Propinare, per ricorrere allo spagn. brindar? La N.C. la registra con un es. del Chiabrera; ma la dice poco usata: poco o punto usata dai ben parlanti, e affatto ignota al popolo, come gli sono ignoti quei banchetti» (Rigutini, 1912: 28; 1926: 28). Cf. DELI, s.v. brindare: secondo il parere concorde degli studiosi dallo sp. brindar (1592). L'uso ottocentesco è imitato dal francese. Per bissare: «Dal bis gridato dagli spettatori quando vogliono ripetuto un pezzo musicale o un ballo, si è fatto dai gazzettieri sciupalingua il verbo Bissare: "Dell’opera furono bissati cinque pezzi". Fino a ieri si diceva Richiedere per rispetto agli spettatori, e Ripetere per rispetto ai cantanti, sonatori e ballerini». (Rigutini, 1912: 26; 1926: 26). 
Preoccupazioni di matrice tipicamente puristica si rintracciano, quindi, nell'avversione rigutiniana per le metafore, che offendono «la gentilezza, la temperanza e l'efficacia antica del parlare italiano con la loro grossolanità, con le enfasi a sangue freddo, e con le incongruenze» ${ }^{18}$. Non è ammesso, infatti, l'uso metaforico dell'aggettivo toccante ${ }^{19}$, che contravviene alla spontaneità, alla schiettezza e alla purezza della lingua ${ }^{20}$. Un lettore chiede se, rispetto all'italianissimo commovente, potrebbe essere considerato come sinonimo con sfumatura diversa di significato. D'altronde anche Dante aveva detto «la vostra miseria non mi tange»: basterebbe questo per giustificare, per analogia, chi lo usa cosi frequentemente? Rigutini risponde confermando l'uso scorretto di questa voce: usare toccare e toccante senza complemento oggetto è «un'ellissi che varrà per il francese toucher e touchant, non così per noi». E, in chiusura di Nota, riferisce che nel Lessico dell'infima e corrotta italianità si legge: «toccante per commovente è il gallicisimaccio (sic) touchant. Fino al gallicismo ci arrivo anche io; non però fino al gallicisimaccio! Mi par d'essere più discreto» (NDL, a. I, n’ 8, 26 marzo 1882).

Un'affermazione che va letta come una dichiarazione di moderazione e, al contempo, una presa di distanza dal radicalismo del Lessico dell'infima e corrotta italianità, che definisce «libro più dannoso che utile agli studj: ma lo combatto impersonalmente e senza pensare a' suoi compilatori, persone degnissime» (Rigutini, 1886).

La posizione moderata del Rigutini, d'altronde, trapela anche da una breve analisi dei titoli delle opere lessicografiche del periodo: accanto al Dizionario de' francesismi [... ] e modi nuovi e guasti di Puoti, al Vocabolario di parole e modi errati che sono comunemente in uso di Ugolini o allo stesso Lessico della corrotta italianità di Fanfani e di Arlia, che aggiunge addirittura infima nel titolo della seconda edizione, quella di Rigutini è l'unica opera che pondera l'esistenza di neologismi buoni.

18. Cf. prefazione all’edizione 1886: «Ma dove è il guasto maggiore e meno rimediabile, è nei nuovi parlari figurati. Questi, mio caro Martini, ci corrompono le fonti del sentimento e del pensiero, da cui scaturisce la lingua, che è poi specchio dell'intima effigie di un popolo. Parla ch'io ti veda, disse quell'antico: lo stesso potremmo dire a una nazione» (Rigutini, 1886: 13).

19. Per la voce toccante cf. NDL a. I, n` 8, 26 marzo 1882. Nell'edizione del 1926 il rimando all'Appendice per la descrizione del lemma è erroneo: il richiamo a p. 171 non ha alcuna corrispondenza, ma coincide con l'Appendice dell'edizione del 1912, in cui si trova ripubblicata la Nota di lingua inerente a toccante (Rigutini, 1926: 161; 1912: 171). In ragione di ciò, quindi, possiamo affermare che l'edizione del 1926 differisce soltanto per l'aggiunta delle voci commentate da Giulio Cappuccini, inserite a fine volume.

20. A riprova di un tipico atteggiamento puristico, nel Lessico di Fanfani-Arlia si deridono quelli che parlano «in punta di forchetta» e, invece, sono viste di buon'occhio le «persone del popolo che chiamano le cose tali e quali / pensatamente schiette e naturali» (Fanfani et Arlia, 1890: 6). 
Contrariamente alla brevità tipica delle Note, Rigutini dedica ben quattro puntate della rubrica al processo della parola risorsa ${ }^{21}$, francesismo riconosciuto e in uso da tempo. In difesa dell'accusata figura il marchese Gino Capponi, accademico della Crusca, che ammette di aver usato il termine in un suo scritto economico. L'avvocato di risorsa dichiara che da Susa a Peloro la voce è accolta da tutti, non c'è strato sociale nella cittadinanza che la rifiuti, dal marchese al trippaio. Una diffusione, quindi, diatopiacamente e diastraticamente capillare. Sennonché alla fine del processo viene giudicata colpevole di indebita naturalizzazione perché «i suoi servigi sono stati accettati dagl'Italiani, parte per loro poltronaggine, parte per malvezzo o per fastidio delle cose nostre» (NDL, a. II, $n^{\circ} 17$, 29 aprile 1883). Rigutini dichiara, però, che la sentenza è stata accolta con mormorio e che non ha soddisfatto nessuno, segno tangibile del fatto che si tratti di una voce ampiamente entrata nell'uso comune e che per questa ragione lo stesso Rigutini, consapevole del naturale processo di evoluzione e arricchimento della lingua, condanna mal volentieri.

Del processo c'è traccia solo nella prima edizione dei Neologismi: nelle edizioni successive verrà sostituito con una più sintetica descrizione ${ }^{22}$ del lemma, come auspicato da Rigutini già nell'Avvertimento del 1886:

Il modo che ho tenuto è breve, sbrigativo, e senza alcuna di quelle lungaggini e insipide pappolate, che hanno rese più che stucchevoli le questioni di lingua tra noi. Per questa ragione vorrei non avere scritto quel Processo della parola Risorsa, anche perché può riescire non gradito a qualche letterato che io grandemente onoro, sebbene discordi dalla sua scuola. (Rigutini, 1886)

La voce, lo sappiamo, resisterà nel tempo nonostante i divieti dei puristi.

21. Per il processo alla parola risorsa, cf. NDL, a. II, $n^{\circ} 14,8$ aprile 1883; n’ 15, 15 aprile 1883; $n^{\circ} 16,22$ aprile 1883; $n^{\circ} 17$, 29 aprile 1883. Come si legge in Serianni (1981: 227), risorsa è presente anche nelle liste dell'Azzocchi: è condannata da Cesari, Bernardoni, Lissoni, Valeriani, Ugolini, Parenti, Bolza, Fanfani-Arlia. La voce è difesa dal Gherardini, che replicò all'elenco del Bernardoni. Bellina (1987: 54) ripercorre la storia moderna del francesismo risorsa: si inizia con i fratelli Verri (1766-1767), quindi la voce dilaga in età napoleonica. Le condanne dei puristi cominciano nel 1798 con Giovanni Antonio Ranza, avvezzo a contrastare la sifilide universale dei gallicismi.

22. Notevolmente ridotto risulta il commento che si trova, perfettamente uguale, nelle altre edizioni: «Risorsa è voce entrata ormai nell'uso comune, quantunque sia tutta di conio francese, e si trasferisce, quel che è peggio, anche a sensi figurati, come: Le risorse dell'ingegno; Le risorse dell'arte, e sim. In molti casi, se non tutti, potrebbero corrisponderle ora Ripresa, ora Provento, ora Rinfranco o Rincalzo, ed ora altre voci. Nella prima edizione di questo libro piacevoleggiai intorno a tal voce con uno scritto intitolato Processo della parola Risorsa, e che in questa seconda non ristampo, fedele a quanto dissi nella Prefazione» (Rigutini, 1912: 142; 1926: 142). Si noti che anche l'edizione del 1926 è indicata come seconda. A questo proposito, si rimanda alla nota riguardante la voce toccante. 
Non è raro rintracciare nelle Note di lingua un certo patriottismo ${ }^{23}$ : alla domanda di un lettore sulla resa in italiano di termini francesi, Rigutini risponde che l'unico modo per dare compiuta espressione ai pensieri è pensare italianamente e l'italiano, a torto ritenuto più povero del francese per la possibilità della lingua d'oltralpe di esprimere più significati in un solo termine, è lingua che ha servito «Dante, Ariosto, Giusti e Manzoni, che ora serve al Carducci ${ }^{24}$ » non può essere la lingua dell'incirca, un surrogato - quindi - del francese. «Sarebbe tempo» - dice Rigutini - «che cessasse questa stupida, e pur troppo antica, glorificazione della lingua della Senna, e che mettessimo un po' il capo a studiare e a conoscere la nostra ${ }^{25}$ \%. Un'idea che ritroviamo in un coevo articolo di Policarpo Petrocchi sulla Domenica Letteraria ${ }^{26}$, che esprime il riconoscimento di una decadenza presente della lingua italiana, comune anche alle opere lessicografiche puriste.

\subsection{Uso toscano}

Di importanza non trascurabile sono le Note in cui Rigutini si sofferma su questioni lessicali riguardanti l'uso vivo toscano.

In una temperie storico-linguistica che vede, accanto alla «scuola della purità e dell'arcaismo intransigente» (Vitale, 1986: 36) iniziata da padre Cesari, altri percorsi, come quello di produzione di un italiano letterario che stava avviandosi a diventare lingua d'uso di una nazione, non sorprende l'attenzione di Rigutini per il toscano contemporaneo. Va ricordato che «l'esistenza di una presunta saldatura tra la lingua degli scrittori trecenteschi e l'uso toscano contemporaneo è un'idea già cir-

23. Sono state espresse riserve sulla genuinità del patriottismo purista e sull'effettiva e convinta adesione al processo risorgimentale da parte dei puristi (Serianni, 1981: 37).

24. Cf. NDL, a. I, n’26, 30 luglio 1882, in cui Rigutini fornisce risposta sui tentativi di tradurre in italiano espressioni come A-t-il de chance!, radoteur, toilette, chaperon, tout-de-même. La Nota è presente anche nell'edizione del 1912, con qualche variante: non c'è il riferimento al Carducci. È possibile che Rigutini, nelle edizioni successive alla prima, abbia maturato una differente consapevolezza delle posizioni ideologiche e linguistiche carducciane, tale da cassare il Carducci dal suo canone degli autori (Rigutini, 1912: 184). La questione affrontata nella Nota non è presente nell'edizione del 1926.

25. A proposito dell'uso di termini stranieri, «la disponibilità all'adozione di elementi forestieri è grande in una nazione che non ha mai avuto confidenza con la propria lingua, in cui il consenso nazionalpopolare non è mai esistito, in cui il sentimento della dignità o potenza della nazione è stato sempre debole, e quando si è sviluppato ha ricevuto il marchio infamante del fascismo» (Marazzini, 2015: 21).

26. Cf. La Domenica Letteraria, a. II, n² 14 gennaio 1883: «[...] non si capisce come gli accenti devano parere pennacchi inutili se non in questo paese dove non abbiamo nessuna cura né della lingua, né della pronunzia [...] e si scrive male perché si parla male e si parla male perché si scrive male: pure rimanendo come siamo, in panciolle e a braccia in croce, aspettando che il tempo accomodi tutto da sé. [... A Allora il mio amico Rissi va a stare in Francia sicuramente. Di dove a parlar sul serio non sarebbe punto male tirare i buoni usi letterari come sinora abbiamo tirato i cattivi. Perché un paese, si rompano pure barriere di monti, di mari, di nemicizie, ma deve e dovrà sempre conservare, se l'ha, il suo carattere; e sarà un delitto di natura guastarlo; e questo va conservato soprattutto nella lingua; per conservarla, va imparata bene; per impararla bene, va regolata meglio, par chiaro; con precisione, direi quasi, perfetta». 
colante nel dibattito sulla lingua» ${ }^{27}$, che incontra, proprio nella seconda metà del XIX secolo, diversi sostenitori anche tra i puristi più defilati o, per meglio dire, non di stretta osservanza.

La Nota di lingua più significativa a proposito dell'uso vivo toscano è una riflessione sul termine pistoiese piallaccio, che - si badi bene - ha luogo solo sulle colonne della Domenica Letteraria e non viene ripubblicata nel volume dei Neologismi.

Rigutini prende spunto da una recensione ${ }^{28}$ alla raccolta di racconti Le veglie di Neri di Renato Fucini, pubblicata a firma di un non meglio identificato B. Il commento del recensore, riportato anche nella Nota, è il seguente:

il toscano del Fucini non appare tratto da un solo vernacolo, ma un po' dall'uno, un po' dall'altro; sicché occorre troppo spesso il non intendere per l'appunto le parole ch' egli usa, e peggio il non aver modo d'intenderle. Lungo la strada da Montecatini a Firenze ho chiesto a parecchi il significato di piallaccio, ma nessuno me l'ha saputo dire. (NDL, a. II, n’30, 29 luglio 1883)

Rigutini ribatte sottolineando come la parola sia comunissima in tutto il pistoiese e in tutta la Val di Nievole e afferma:

Che cosa adunque sia il piallaccio glielo farò dire a Cosimo Trinci pistoiese, e scrittore di Crusca, nel seguente esempio (Agric. 199): «Né si lascino di fare in più luoghi delle coltivazioni i suoi muretti a secco, ovvero cigli di piallacci, o, come altri dicono, piote di terra erbata.» Piallaccio dunque ha capito che cos'è: è piota di terra erbata. Il Fucini usandolo in due luoghi delle sue bellissime Veglie, non usa certamente una voce di gergo. (NDL, a. II, n’30, 29 luglio 1883)

Un termine, dunque, rintracciabile nella lingua della tradizione cruscante ${ }^{29}$ ed un richiamo da parte di Rigutini all'uso degli scrittori - su questo aspetto torneremo dopo - che assolve Fucini dall'accusa di usare «una voce di gergo» (NDL, a. II, n’30, 29 luglio 1883) e che, nella sostanza, fa trasparire la preoccupazione, tutta puristica, di evitare i tecnicismi settoriali e i dialettismi.

Il lessicografo continua poi ad interrogarsi sull'origine di questa parola e si richiama al Dizionario d'agricoltura e pastorizia di Palma, in cui compare pelliccia nel significato di «pezzo di terra erbosa che si leva dal prato», voce che, secondo Rigutini, nell'uso si scambia con piallaccio, proprio perché quest'ultima è una forma corrotta di pelliccia:

27. Cf. Picchiorri (2008: 54), che evidenzia la posizione assunta da Bresciani, oscillante tra neotoscanismo naturalistico e purismo.

28. La recensione è apparsa sul periodico La Cultura. Cf. B. (1883), «Le veglie di Neri. Paesi e figure della campagna toscana», La Cultura, anno II, vol. 4, n 6, 15 giugno 1883, p. 474-476.

29. Cf. GDLI, s. v. piallaccio: nell'accezione di zolla di terra erbosa, le uniche attestazioni sono di Trinci e Fucini. 
Odasi il Palma (Diz. D’Agricoltura e Pastorizia, Milano 1870): «Pelliccia vale anche Pezzo di terra erbosa che si leva dal prato e dal campo come una crosta: ha senso identico a Piota, sicché nell'uso si scambiano. Con le pellicce o piote si rivestono gli argini o ciglioni dei campi di collina e simili. Da Pelliccia si è fatto Impellicciare, che vale coprir di pellicce». Ma i vocabolari della lingua comune, dirà il B., non danno né Piallaccio, né Pelliccia, né Impellicciare in questo senso: o di dove li cavate voi? Di dove? Dall'uso, dinnanzi al quale i vocabolari son sempre corti. Ciò non ostante, io non approvo che nel vocabolario si registrino voci o forme diverse da quelle che sono in uso nel centro della lingua, come nel nostro caso sarebbe Piallaccio [...]. (NDL, a. II, n’30, 29 luglio 1883)

Ingiusto gli appare, quindi, il rimprovero mosso al Fucini: «non è giusto far rimprovero a uno scrittore pistojese se adopera voci della sua provincia e in racconti la cui scena è colà; e tanto meno è giusto metterlo quasi in voce di scrittore di gergo».

Per difenderlo ulteriormente dall'accusa di usare troppi pistoiesismi, Rigutini riporta le parole di Giovanni Procacci, autore della prefazione al volume Le veglie di Neri, il quale contesta al Fucini unicamente «d'essere poco audace nell'appropriarsi le bellezze vive del parlare toscano, e [...] le vigorose locuzioni di quel territorio pistoiese che egli corre ogni giorno per lungo e per largo come cacciatore e come ispettore scolastico».

A commento di questa citazione, il lessicografo rileva come gli scrittori, divisi tra coloro che li accusano di usare troppi toscanismi e quelli che li rimproverano di usarne pochi, non sappiano a quale santo votarsi e denuncia in Italia un clima di incertezza a proposito di «criteri sull'arte, sulla lingua e su qualche altra cosa». Un'esternazione che dimostra quanto fosse vivo il dibattito sulla «emarginazione della lingua letteraria» (Gatta, 2014: 323) e della prosa arcaica a favore della legittimità dell'uso, specchio della lingua viva dei parlanti. Quella di Rigutini si configura, dunque, come una piena difesa del Fucini, che accoglie nelle sue pagine toscanismi marcati - e in parte estranei all'uso fiorentino che raramente si troverebbero nella prosa media manzoniana dei Promessi Sposi. È una posizione che colloca ancor di più Rigutini nel solco del purismo, fedele all'ideale linguistico dell'aureo Trecento da rinverdire con la viva voce dei popolani toscani, avvezzi ad usare ancora termini riconducibili ai loro illustri antenati.

L'interesse per i toscanismi, la lingua parlata e le sue varietà (basterebbe già citare il noto Vocabolario della lingua italiana parlata di Rigutini-Fanfani del 1875) si manifesta anche nella scelta di dare spazio ${ }^{30}$ al quesito di un lettore riguardante una «parola che indichi esattamente quella specie

30. In NDL a. I, n 12, 23 aprile 1882 aveva scritto: «vorrei essere cortese di risposta a tutti coloro, i quali attribuendomi molto maggiore autorità che non sento d'avere, mi rivolgono domande di lingua. Ma come non potrei senza occupare troppo spazio nella Domenica Letteraria, così mi veggo costretto a dichiarare, che alcune di queste domande avranno a suo luogo la risposta nelle Note susseguenti, e che ad altre non posso rispondere, perché concernenti certi parlari figurati che bisogna lasciare al gusto, corretto e scorretto, di chi le adopra». 
di vernice argentea che ricopre le susine ${ }^{31}$. . Ciò dimostra che Rigutini è sensibile ai livelli diafasici della lingua, passando dal letterario all'usuale. Il lettore specifica che «nel pisano si usa rezzola» e, nel senso di «lieve pellicola che ricopre l'aglio e le cipolle», viene anche citata da Fanfani in un suo opuscolo. Lo studioso risponde:

nel fiorentino dicesi comunemente fiore, nel senese pruina; né so se in altre provincie toscane si usi diverso vocabolo. Ma questi due a me paiono assai belli; [...] il secondo è più proprio e più significativo, poiché quel pulviscolo ti dà appunto l'idea della brina o pruina posatasi sul frutto. [...] II latino pruina passando nell'italiano si mutò in brina, rimase poi nel parlare del senese a significare [...] soltanto quella data cosa. (NDL, a. II, n’31, 5 agosto 1883)

Una risposta che dà dignità e profondità storico-linguistica alla voce senese, eletta dal lessicografo come vocabolo più appropriato per esprimere il concetto richiesto dal lettore.

In una Nota precedente, invece, il Rigutini aveva risposto a lettori non toscani che si interrogavano sulla locuzione toscana tornar di casa: «[...] come mai si dica Tornar di casa in un luogo, quando in quella casa non si è mai stati»; per spiegarla, si rifà al significato primitivo del verbo tornare:

il verbo Tornare conserva nella maniera toscana il senso che ebbe primitivamente, ed in comune con altre lingue romanze, in alcune delle quali, come nella francese, è tuttora rimasto, il senso dico di volgere o volgersi, affine al latino versare e versari32; onde poi le conseguenti idee di mutazione, cangiamento da uno stato, condizione e simili in un’altra. Dante dice: Ché dalle reni era tornato il volto (Inf. 20): dalla parte delle reni quegli spiriti non ce l'avevano mai avuto; il Cavalca dice che la moglie di Lot tornò in istatua di sale: quella donna di sale non era mai stata. (NDL, a. I, n’ 30, 27 agosto 1882)

Cita Dante e il Cavalca per dimostrare come il verbo tornare denoti un rivolgimento o un cambiamento e conclude dicendo che per i toscani tornar di casa in un luogo significa volgersi ad una nuova abitazione, ma «questo stesso verbo fu usato fin quasi al seicento per abitare, alloggiare». A questo proposito riporta tre citazioni: una del Trecento, una del Quattrocento e una del Cinquecento ${ }^{33}$. Ancora uno stuolo di esempi da scrittori della tradizione letteraria per giustificare «questa maniera toscana oggi vivissima», che - continua Rigutini - «dopo questa noterella, confido non sia per sembrar più né strana né degna di riso». In un'altra Nota ${ }^{34}$, volge la sua attenzione a modi proverbiali

31. Le Note riguardanti rezzola e la locuzione tornar di casa si trovano anche nell'edizione del 1912 (Rigutini, 1912: 196; 1912: 180). Cf. GDLI, s.v. rezzola: buccia sottile della cipolla; anche strato superficiale di panna che si forma sul latte: in questa accezione è attestato nel Vocabolario pistoiese.

32. Si noti il riferimento al verbo greco topveúw, aggiunto, dopo il latino versare e versari, nell'edizione 1912 (Rigutini, 1912: 181).

33. Rigutini scrive: «per chi ama le citazioni eccone qui tre: [...] dalla Storia di Apollonio (12), da Vespasiano da Bisticci (Vit. 154) e da Benedetto Varchi (Stor. I, 619)» (NDL, a. I, n’30, 27 agosto 1882).

34. Cf. NDL, a. II, n’29, 22 luglio 1883, ripubblicata nell’edizione del 1912 (Rigutini, 1912: 178). 
toscani «significanti tutti in gran copia, in gran quantità»: a biscia, a isonne, a macca, a bizzeffe. Prova a ricostruirne l'etimologia: per a biscia ${ }^{35}$ propone la voce bica, mentre «a isonne ${ }^{36}$ forse, come nota il Tommaseo, è scorcio di Eleisonne, perché ripetesi tre volte nelle litanie». A proposito di bizzeffe, avanza dubbi sulla validità delle sue ipotesi: «se non èvera, è ben trovata», mentre si dice sicuro che $a$ macca derivi da macco, che rileva nel Morgante del Pulci. In chiusura di Nota, suggerisce al suo egregio amico Morandi di raccogliere «tutte le maniere, di cui è ricchissima la nostra lingua, significanti idea di grande abbondanza» poiché «sarebbe il solo profitto che potrebbe uscire da questa Nota».

\section{Conclusioni}

Abbiamo fatto riferimento più volte all'uso degli scrittori e al richiamo alla tradizione letteraria.

Nella prefazione ai Neologismi del 1886, Rigutini afferma che «tutta l'autorità e ogni criterio filologico è posto nei parlanti» e si dichiara grato a Manzoni «il quale, tolta la questione ai pettegolezzi e alle piccinerie dei grammatici e inalzatala a questione nazionale, pose risolutamente nell'uso parlato, sia pure di una sola città toscana, il pernio dell'idioma» (Rigutini, 1886: 11). Si può parlare, in questo senso, di un'adesione teorica del lessicografo ai principi manzoniani (da inserire, però, nel quadro di una scoperta dell'uso vivo toscano che anche i puristi di più larghe vedute, come Bresciani, avevano praticato), più che di una realizzazione linguistica effettiva delle teorie manzoniane, sulla quale sarebbe impossibile indagare in maniera esaustiva in base agli elementi che il nostro studio, limitato alle Note di lingua, ci fornisce.

Pare certo, però, che il concetto di uso maturato da Rigutini rivendichi ancora la funzione degli scrittori nella regolamentazione della lingua o nella legittimazione di certi usi linguistici ${ }^{37}$. Diverse erano, invece, le intenzioni del Manzoni, legato all'idea di uso inteso come «vita della parola in una vera comunità di parlanti» (Marazzini, 2002: 381). Per Rigutini occorre, invece, confrontare la lingua parlata con la scritta e rinfrescare la lingua scritta «coi ruscelli vivi e perenni che derivano dalla bocca del popolo» (Rigutini, 1912: XV): in questo ci sembra che l'ideologia del lessicografo possa configurarsi come anello di congiunzione tra purismo e manzonismo.

35. Cf. TB, s.v. biscia: a biscia per in copia non viene forse dalla fig. dell'animale, ma dal suono imitato come di liquore che scorre in gran copia. Nel GDLI, s.v., attestazioni in Machiavelli, Redi, e in Note al Malmantile.

36. Cf. Fanfani (1863), s. v. isonne: modo basso. Nel GDLI, s.v., attestazioni abbondanti dalla tradizione toscana, tra cui Giusti. Etimo incerto, cf. Note al Malmantile.

37. «In questa seconda edizione ho aggiunti gli esempi degli scrittori autorevoli per difendere l'uso di certe voci o locuzioni; poiché il solo citarli non basta, pochi avendo avendo comodità di riscontrarli nei grandi Vocabolari, e specialmente in quello dell'Accademia della Crusca» (Rigutini, 1912: VI). 
Un altro richiamo al Manzoni è compiuto da Rigutini a proposito dell'«accettare quanto non discorda dall'orecchio e dal sentimento italiano» ${ }^{38}$, cioè accogliere quei forestierismi che si mantengono nell'alveo della struttura fonomorfologica dell'italiano. Si intende, quindi, assecondare il naturale processo evolutivo della lingua e convincersi che la norma non scaturisce dalle prescrizioni, ma dalla coscienza linguistica dei parlanti. Posizioni ideologiche, queste, che ci fanno pensare al neopurismo di Migliorini.

Specchio e proiezione del mutamento di percezione in atto, introdotto da Rigutini rispetto alle rigide posizioni dei censori puristi, è all'inizio del Novecento l'atteggiamento di un altro noto lessicografo, Alfredo Panzini, che, nel suo Dizionario moderno del 1905, si limiterà a registrare una realtà linguistica in continuo cambiamento.

38. Cf. Avvertimento alla prima edizione (Rigutini, 1886), in cui il lessicografo rimarca la sua adesione teorica ai principi manzoniani. 


\section{Bibliografia}

\section{Fonti primarie}

Rigutini, Giuseppe (1886), I Neologismi buoni e cattivi più frequenti nell'uso odierno, Roma, Verdesi.

Rigutini, Giuseppe (1905), I Neologismi buoni e cattivi più frequenti nell'uso odierno, Firenze, Barbera.

Rigutini, Giuseppe (1912), I Neologismi buoni e cattivi più frequenti nell'uso odierno, Firenze, Barbera.

Rigutini, Giuseppe (1926), I Neologismi buoni e cattivi più frequenti nell'uso odierno, Nuova edizione con prefazione e aggiunte di Giulio Cappuccini, Firenze, Barbera.

\section{Fonti secondarie}

Bellina, Massimo (1987), «Girolamo Tagliazucchi, Luigi Angeloni e le origini della lessicografia puristica ottocentesca», Studi linguistici italiani, n’13, p. 40-62.

Bellina, Massimo (2007), «Sull'epistolario di Antonio Cesari, con una lettera inedita a Luigi Angeloni e alcune note sul purismo», in Della Valle, Valeria e Pietro Trifone (ed.), Studi linguistici per Luca Serianni, Roma, Salerno, p. 51-72.

DELI: Cortelazzo, Manlio e Paolo Zolli (1999), Dizionario Etimologico della lingua italiana, Bologna, Zanichelli.

Della Valle, Valeria (1993), «La lessicografia», in Serianni, Luca e Pietro Trifone (ed.), Storia della lingua italiana, vol. 1, Torino, Einaudi, p. 72-74.

De Martini, Silvia (2014), Grammatica e grammatiche in Italia nella prima metà del Novecento, Firenze, Cesati.

De Roberto, Elisa (2016), “"A scriver come si parla si guadagna un tanto”. Ida Baccini e l'insegnamento dell'italiano», in Pierno, Franco e Giuseppe Polimeni (ed.), L'ttaliano alla prova. Lingua e cultura linguistica dopo l'Unità, Firenze, Cesati, p. 91-115.

Fanfani, Pietro (1863), Vocabolario dell'uso toscano, Firenze, Barbera.

Fanfani, Pietro e Costantino Arlìa (1890), Lessico dell'infima e corrotta italianità, Milano, Carrara. [1 $1^{\circledR}$ ed., 1881]

Franceschini, Fabrizio (2011), «I nipotini di Padre Cesari: il purismo e la sua influenza nella scuola dell'Italia unita», in Nesi, Annalisa, Silvia Morgana e Nicoletta Maraschio (ed.), Storia della lingua italiana e Storia dell'Italia unita. L'italiano e lo stato nazionale. Atti del IX Convegno Asli, Firenze, Accademia della Crusca, 2-4 dicembre 2010, Firenze, Cesati, p. 295-309.

Gatta, Francesca (2014), «Giornalismo», in Antonelli, Giuseppe, Matteo Motolese e Lorenzo Tomasin (ed.), Storia dell'italiano scritto, vol. 3, Roma, Carocci, p. 293-347. 
GDLI: Grande dizionario della lingua italiana (1961-2002), 21 vol., fondato da Salvatore Battaglia, poi diretto da Giorgio Bàrberi Squarotti, Torino, Utet.

Librandi, Rita (2014), «Ancora su Giuseppe Bernardoni, corrispondente di Monti, librettista e purista per caso», Lingua e Stile, n’49, p. 237-265.

Marazzini, Claudio (2002), La lingua italiana. Profilo storico, Bologna, il Mulino.

Marazzini, Claudio (2015), «Perché si è tanto propensi ai forestierismi?», in Marazzini, Claudio e Alessio Petralli (ed.), La lingua italiana e le lingue romanze di fronte agli anglicismi, Firenze, Accademia della Crusca, p. 14-26.

Milani, Celestina (1978), «Tra lingua e invenzione: un lessico del 1862», Italianistica, n 7, p. 85-98.

Perugini, Marco (2013), «"I gentili mantenitori di nostra lingua”: Marc'Antonio Parenti e il purismo di provincia», in Tomasin, Lorenzo (ed.), II Vocabolario degli Accademici della Crusca e la storia della lessicografia italiana. Atti del X Convegno Asli, Padova, 29-30 novembre 2012 - Venezia, 1 dicembre 2012, Firenze, Cesati, p. 225-236.

Picchiorri, Emiliano (2008), La lingua dei romanzi di Antonio Bresciani, Roma, Aracne.

Rigutini, Giuseppe (1879), Vocabolario della lingua italiana compilato per uso delle scuole accresciuto di voci del comun parlare e degli approvati scrittori, Firenze, Barbera.

Serianni, Luca (1981), Norma dei puristi e lingua d'uso nell'Ottocento, Firenze, Accademia della Crusca.

Serianni, Luca (2006), «Panzini lessicografo tra parole e cose», in Adamo, Giovanni e Valeria Della Valle (ed.), Che fine fanno i neologismi?, Firenze, L. S. Olschki, p. 55-78.

Serianni, Luca (2013), Storia dell'italiano nell’Ottocento, Bologna, il Mulino.

TB: Tommaseo, Niccolò e Bernardo Bellini (1865-1879), Dizionario della lingua italiana, Torino, Unione Tipografico-Editrice.

Vinciguerra, Antonio (2015), Purismo e antipurismo a Napoli nell'Ottocento, Firenze, Società Editrice Fiorentina.

Vitale, Maurizio (1984), La questione della lingua, Palermo, Palumbo.

Vitale, Maurizio (1986), L'oro nella lingua, Milano-Napoli, Ricciardi.

Zolli, Paolo (1974), Saggi sulla lingua italiana dell'Ottocento, Pisa, Pacini. 